\title{
Method for determining the potential strain energy stored in the earth before a large earthquake
}

\author{
E. E. Khachiyan \\ Institute of Geological Sciences, National Academy of Sciences, Prosp. Marshal Bagramyan, 24a, Yerevan, 0019, Republic of Armenia
}

Email address:

edkhach@sci.am (E. E. Khachiyan)

\section{To cite this article:}

E. E. Khachiyan. Method for Determining the Potential Strain Energy Stored in the Earth before a Large Earthquake. Earth Science. Vol. 2, No. 2, 2013, pp.47-57. doi: 10.11648/j.earth.20130202.14

\begin{abstract}
This paper describes a technique for determining the potential energy of deformed material around a future earthquake rupture, with this energy being stored during the precursory period. The basic parameters are the following: rupture length on the Earth's surface after the earthquake has occurred $L$, rupture depth $\mathrm{h}$, and the relative block movement along the rupture strike line $\bar{u}$. We compared the results for 44 large earthquakes with those derived by determining seismic wave energy from earthquake magnitude.
\end{abstract}

Keywords: Earthquake; Strain Energy; Energy Seismic Waves; Comparison

\section{Introduction}

According to current concepts [1-5], an earthquake results from a mechanical rupture in the earth due to the contact of two geological blocks (plates) with uneven edges as they slowly move in opposite directions. Prior to the earthquake, a region in the contact medium is under continually growing mechanical stresses due to deformation of the rocks around the rupture; hence, an amount of energy is stored there. This is the potential energy of the medium or strain energy As a matter of fact, this energy is the total (complete) energy that has been stored in the earth before the earthquake. This complete energy separates on being released by the earthquake into the energy of seismic waves (kinetic energy) and thermal friction energy on the responsible fault, or into the energy required to crush the host rocks [1].

There are several relations [6] in use for determining the energy of seismic waves. We used the following relation [7]:

$$
\log E_{S}=11.8+1.5 M_{S}, \quad k_{S}=\log E_{S}
$$

where $E_{S}$ is the energy of seismic waves in ergs, $M_{S}$ is earthquake magnitude, and $k_{S}$ is the earthquake energy class when $E_{S}$ is in Joules.

There is no well-established theory in seismology to derive quantitative relationships between the energy of seismic waves and the thermal energy of fault friction. Much depends on the coefficient of sliding friction. The ratio between the two types of energy for different earthquakes must in principle be different. The ratio strongly depends on the mechanism of rock rupture and on the orientation of the rupture plane. Several researchers [8-10] believe that melting must occur on the rupture plane if the friction stress (of blocks) during a large earthquake exceeds a definite threshold. However, there is scant mention of such phenomena in the literature. The present study develops a simple method for determining the potential energy in a medium that has been deformed during the precursory period of a large earthquake based on macro seismic impact on the Earth's surface after the event.

\section{Formulation of the Problem}

In a general case the potential energy stored in an elastic medium, $E_{d}$, can be expressed as follows $[1,11]$ :

$$
\begin{aligned}
E_{d}=\frac{1}{2} \int_{V}\left(\sigma_{x x} \varepsilon_{x x}+\sigma_{y y} \varepsilon_{y y}+\sigma_{z z} \varepsilon_{z z}+\right. \\
\left.+\tau_{x y} \gamma_{x y}+\tau_{y z} \gamma_{y z}+\tau_{x z} \gamma_{x z}\right) d V
\end{aligned}
$$

where $\sigma_{i i}, \tau_{i j}$ and $\varepsilon_{i i}, \gamma_{i j}$ are the tensors of normal and tangential stresses and strain, respectively and $\mathrm{V}$ is the total volume of the medium under stress and strain.

One essential drawback to (2) is that the expression for $E_{d}$ is the elastic energy of a medium not under stress previously. This formulation of the problem is actually consistent with the Reid model for earthquake generation where "during an earthquake the two sides of the fault experience a 
mutual movement that exactly corresponds to the complete release of elastic stress on the fault" [12]. However, the stress in the medium is not released completely by every earthquake. Actually, the precursory process of an earthquake starts in the medium under some initial stresses $\sigma_{i j}^{0}$, and, as a consequence, with some initial elastic energy $E_{0}$. The energies $E_{d}$ and $E_{0}$ are not additive; hence, the correct expression for total elastic energy will involve cross terms of the form $\sigma_{i j}^{0} \varepsilon_{i i}$, where $\varepsilon_{i j}$ are the strain components of the imminent earthquake. According to many specialists, the character of the distribution and the values of the initial stresses are primarily related to the presence of secondary ruptures and various cracks in the rupture zone. What exactly is the stress that has been released by an earthquake can be found by measuring the associated strains before and after the earthquake. Such measurements have been made for several earthquakes.

The influence of initial stresses on the final value of potential energy stored as strain in the medium before an earthquake is very likely determined by the start time and duration of the precursory period. There may be cases in which the residual stresses of a previous earthquake have completely or partially disappeared before the precursory period of the next earthquake. No clear-cut answers to such questions are available in the literature in earthquake mechanics. Simple calculations that we carried out based on [1] show that, for a small volume $\Delta V$ in the central part of the rupture where the stress field is approximately oriented in the fault plane before and after the earthquake, one can get

$$
\Delta E=\Delta E_{d}\left(1-\frac{\tau_{0}^{2}}{\tau^{2}}\right)
$$

where $\Delta E$ is total energy with initial stresses incorporated, $\Delta E_{d}$ is the energy without initial stresses, $\tau_{0}$ is initial tangential stress, and $\tau$ is the tangential stress before the earthquake. As can be seen from (2a), the ratio $\Delta E / \Delta E_{d}$ varies in a narrow range (between 1 and 0.91 ) when $0 \leq \tau_{0} / \tau \leq 0.30$; this ensures that the use of (2) cannot entail serious error in many cases. We also note one important circumstance that arises in the use of (2): all calculations of $E_{d}$ from (2) directly involve, not the stress values, but the actual slip $\bar{u}$ and the geometrical characteristics of the new rupture $L$ and $h$ resulting from an earthquake, which cannot arise without the influence of initial stresses in the medium. It is difficult to predict whether this can happen completely or partially. By the way, neither does the determination of earthquake energy using the empirical relation
(1) directly employs the effects of initial stresses, since earthquake magnitude $M_{S}$ is found from actual characteristics of ground motion (trace amplitude and period) of seismograms during the earthquake.

In view of the above, the question as to what exactly the potential strain energy stored in the medium before a large earthquake obtained by (2) is remains open.

We now formulate the following problem: Find the total potential energy (2) after an earthquake from its effects on the Earth's surface: rupture length $L$, fault depth $h$, and relative slip along the fault $u$. Figure 1 shows a schematic distribution of stress and strain before and after a rupture and the distribution of tangential stresses near the rupture in the direction perpendicular to the rupture, where $\tau_{T}$ is the tangential stress yield limit.

It is natural, to a first approximation, to assume that the stress distributions to the left and to the right of a future rupture are identical in character. The most important parameter of these regions is the extent $H$ to which tangential stresses penetrate in the direction perpendicular to the rupture.

We will assume that the state of stress and strain around a future rupture on the Earth's surface has the form shown in Fig. 1 as the shaded region confined within a closed curve $C$. We also assume that the stresses and strains in the medium outside the region $C$ are small compared with their values near the rupture; hence, they can be neglected in the determination of total potential energy. It can be seen from this figure that the region of incorporated stresses and strains confined within $C$ can be replaced with an equivalent rectangular area with sides $2 H$ and $L$ shown by dashed lines. In other words, we assume that the state of stress and strain in the earth before the occurrence of an earthquake was concentrated in two rectangular parallelepipeds with sides $L, H$, and $h$, where $h$ is rupture depth (block size in depth, Fig. 1b), $L$ is the length of the actual rupture on the Earth's surface, and $H$ is the distance from the rupture beyond which the earth can be treated as being free of the stresses that were induced by the future earthquake.

We assume that during the last phase in the earthquake precursory process each of the two parallelepipeds has a slip equal to $u / 2$, while after the rupture it will have a relative slip of $u$ (see Fig. 1b). The relative slips along the rupture usually obey a no uniform distribution. For this reason it is advisable to take some mean value $\bar{u}$ as the slip for the entire rupture $L$.

For several large earthquakes the quantity $\bar{u}$ was determined by Wells and Coppersmith [13] who believe that it is in relation to the mean slip $\bar{u}$ that the relative slip can be assumed to be identical throughout the length and depth of paired fault planes. 

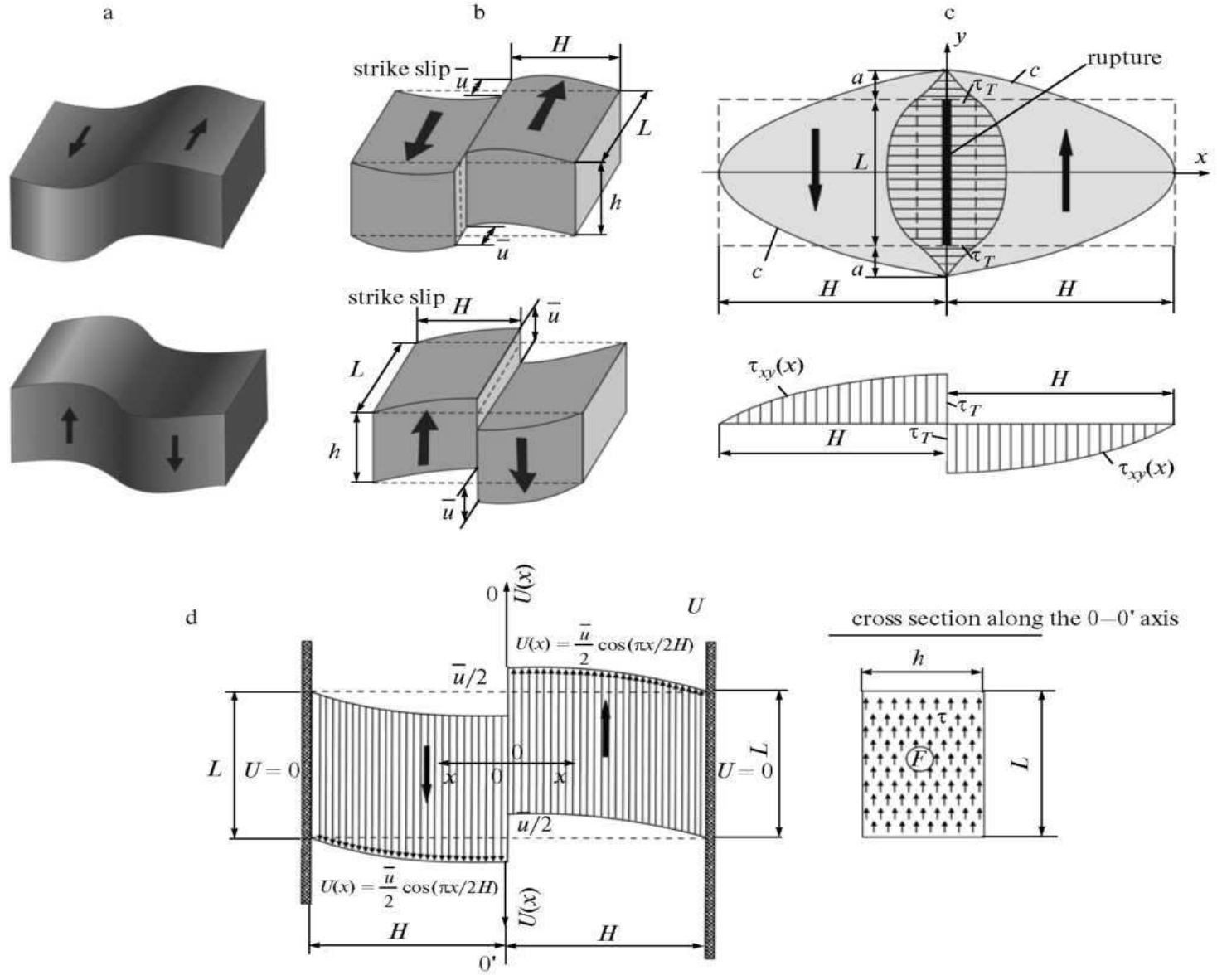

cross section along the $0-0^{\prime}$ axis

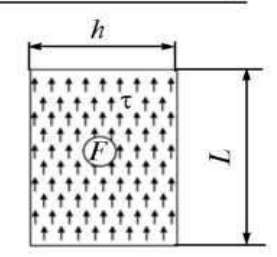

Fig. 1. Schematic presentation of the state of stress and strain in the medium (a) schematic picture of strain before rupture; (b) after rupture; (c) shear stress distribution; (d) theoretical strain diagram after rupture. L is rupture length, $h$ rupture depth, $H$ the extent of strain region perpendicularly to the rupture, $u$ the mean relative slip, $F$ fault area, $\tau_{T}$ strength limit, a the extent of strain region parallel to the fault, $C$ the curve that confines the strain region, $u(x)$ the slip function perpendicularly to the fault. Arrows show the directions of slow block movement.

\section{Boundaries of Deformed Regions Around the Rupture}

Now we consider the question of how to find the regions of felt strain during the precursory periods of earthquakes until the very last phase that culminates in rupture. Reid [12] was the first to use measurements of this strain from geodetic triangulations conducted before and after the California earthquake of 1906.

Many such plots for tens of Japanese and American earthquakes can be found in [14], a work devoted to earthquake prediction.

Figure 2 shows such relations for three earthquakes from the above book. These plots give some idea of the quantitative decay of strain magnitude with distance from the fault perpendicular to it. Based on these and other similar results quoted in this work we propose the following empirical relation between the depth of strain $\mathrm{H}$ (in meters) and mean slip $\bar{u}$ (in meters):

$$
H=(5 \bar{u}+15) \times 10^{3},
$$

According to (3), assuming the mean relative slip of the blocks to be $\bar{u}=1 \mathrm{~m}$, we find that the ground displacement before the rupture and after the earthquake rupture must be set equal to zero for a distance $H=10^{3}(5 \times 1+15)=20 \mathrm{~km}$ from the rupture to both sides of it throughout the entire rupture length $L$, that is, outside the area $L \times 2 H$, and $H=25 \mathrm{~km}$ for $\bar{u}=2 \mathrm{~m}$. Relation (3) is applicable to large earthquakes only with a relative surface slip of at least 10-1 $\mathrm{m}$ in order to make the error of measurement in the field negligible. It can naturally be supposed that relation (3) given here might produce results for some individual earthquakes that are much different from the recorded actual results.

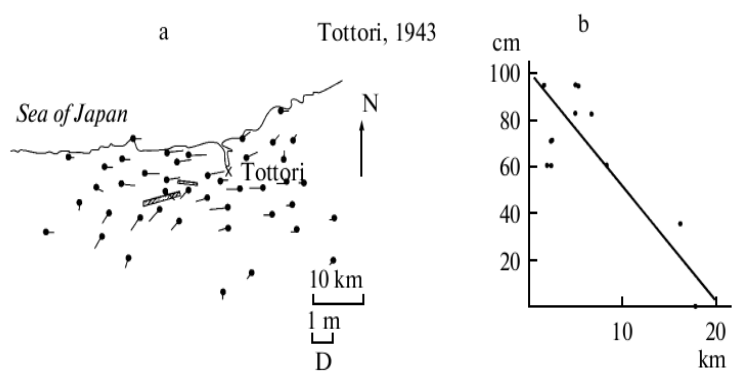




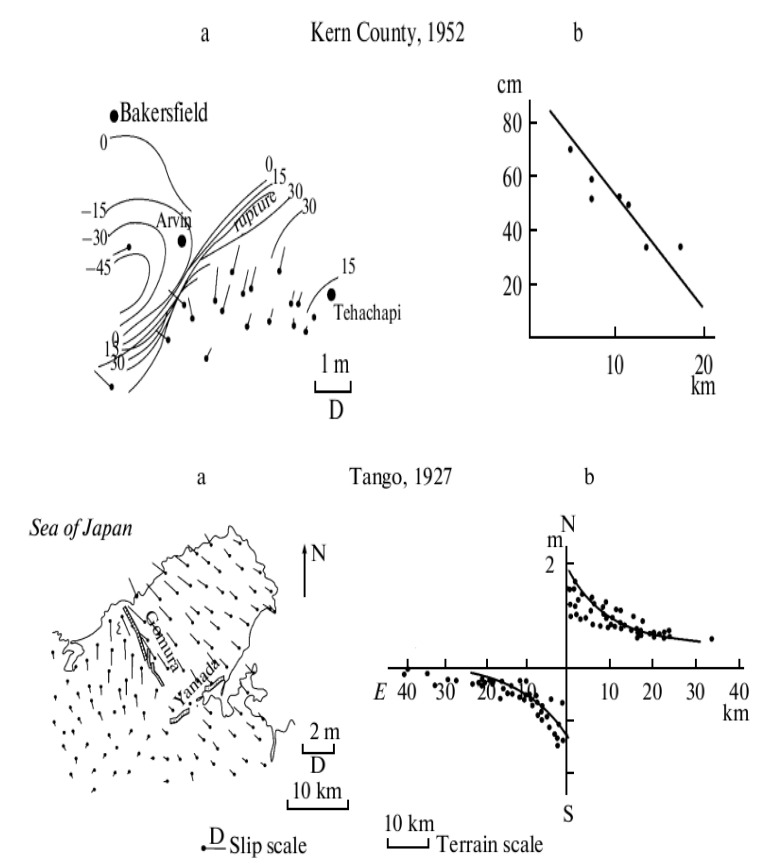

Fig. 2. Displacements of triangulation stations and the falloff of their values. (a) horizontal displacements of triangulation stations caused by the Tottori, Kern Country, and Tango earthquakes [14]; (b) falloff of horizontal displacements parallel to the fault with the distance to the fault [14]

Corrections to such relationships could be obtained from solutions of theoretical problems by methods of elasticity theory. Kasahara [4] used solutions to problems in elasticity theory for the model of a vertical fault with strike separation in the form of an infinite strip that intersects the surface of an elastic half-space under homogeneous shearing stresses [15] to give the theoretical relationship between the slip $\bar{u}$ on the Earth's surface along the direction of the fault strike and the coordinate $x$ perpendicular to the fault, as follows:

Table 1. Distance $\mathrm{H}$ in relation to rupture depth $\mathrm{h}$ and a coefficient $\alpha$

\begin{tabular}{llll}
\hline$h \times 10^{-3}, \mathrm{~m}$ & $\begin{array}{l}H \times 10^{-3}, \mathrm{~m} \\
\alpha=0.1\end{array}$ & $\alpha=0.15$ & $\alpha=0.2$ \\
\hline 10 & 49 & 32 & 24 \\
15 & 74 & 49 & 36 \\
20 & 98 & 64 & 48 \\
\hline
\end{tabular}

$$
u(x)=\frac{u_{\max }}{2}\left[\sqrt{(x / D)^{2}+1}(-x / D)\right]
$$

where $u_{\max }$ is the peak (maximum) amplitude of slip and $D$ is the vertical extent of the fault (fault depth $h$ ). The maximum slip $u_{\max }$ is related to the other parameters as follows:

$$
u_{\max }=\frac{2 \Delta \sigma D}{G}, \Delta \sigma=\frac{1}{2} \frac{G u_{\max }}{D}
$$

where $\Delta \sigma$ denotes the stress drop by the rupture and $G$ is the shear modulus.

Relation (4) can be used to estimate $H$, the depth to which felt strain penetrates. For example, if we limit ourselves to the case of a half-space where slips below $\alpha u_{\max }(\alpha<1)$ are treated as virtually non-existent (here, $\alpha$ is the ratio of slip as given by (4) at a distance $x=H$ from the fault $u(H)$ to the maximum slip on the fault $u(0)$ at point $x=0)$, then we get from (4).

$$
\frac{u_{\max }}{2}\left[\sqrt{\left(\frac{H}{D}\right)^{2}+1}-\frac{H}{D}\right]=\frac{\alpha u_{\max }}{2},
$$

from which the expression for $H$ is,

$$
H=\frac{1-\alpha^{2}}{2 \alpha} D
$$

With $\alpha=0.2$ and fault depth $D=h=10 \mathrm{~km}$, we get $H=24 \mathrm{~km}$ from (6). The values of $H$ for various $\alpha$ and $h$ are given in Table 1 .

The appendix to K. Kasahara's work mentioned above also contains a formula (for Japan) to determine the radius of the deformed area $r(\mathrm{~km})$ in relation to earthquake magnitude $M$ [4] as follows:

$$
\log r=0.51 M-2.27
$$

which can also be used to provide an idea of the abovementioned extent $H$ of the incorporated strain. According to this formula, we get $r=H=6 \mathrm{~km}, 20 \mathrm{~km}$, and $64 \mathrm{~km}$ for earthquakes with magnitudes $M=6,7$, and 8 , respectively.

\section{Potential Strain Energy}

Having now at our disposal the values of mean slip $\bar{u}$ rupture length $L$, rupture depth $h$, and the extent of the region of incorporated strain $H$, we are in a position to find the potential energy from (2). Assuming the region $L \times 2 H$ to be under pure shear, we shall have [11]:

$$
\begin{gathered}
\sigma_{x x}=\sigma_{y y}=\sigma_{z z}=0 \quad \tau_{x y}=\tau(x), \quad \tau_{y z}=\tau_{x z}=0 \\
\gamma_{x y}=\frac{\partial u}{\partial x}, \quad \gamma_{y z}=\gamma_{x z}=0 \\
\tau(x)=G \frac{\partial u}{\partial x}, \quad d V=L h d x \quad 0 \leq x \leq H .
\end{gathered}
$$

Consequently, the total potential energy will be

$$
E_{d}=2 \frac{1}{2} \int_{0}^{H} \tau(x) \frac{\partial u}{\partial x} L h d x=\frac{2 L h G}{2} \int_{0}^{H}\left(\frac{\partial u}{\partial x}\right)^{2} d x .
$$

For $u(x)$ we will use a simpler formula:

$$
u(x)=\frac{\bar{u}}{2} \cos \frac{\pi x}{2 H}
$$

which satisfies the boundary conditions of the problem 
(see Fig. 1d):

$$
\begin{array}{ll}
\text { for } x=0 & u=\frac{\bar{u}}{2} \\
\text { for } x=H & u=0
\end{array}
$$

Using (10), we get for potential energy $E_{d}$ :

$$
E_{d}=2 \int_{0}^{H} \frac{L h G}{2} \frac{\bar{u}^{2}}{4} \frac{\pi^{2}}{4 H^{2}} \sin ^{2} \frac{\pi x}{2 H} d x
$$

Doing the operation of integration, we derive the following simple relation:

$$
E_{d}=\frac{\pi^{2}}{32} \frac{L h G}{H} \bar{u}^{2} \text { or } E_{d}=0.308 \frac{L h G}{H} \bar{u}^{2}
$$

The value of energy $E_{d}$ in (9) was calculated using the decaying slip function $u(x)$ from (4). In that case we shall have for $E_{d}$ :

$E_{d}=\frac{2 L h G}{2} \int_{0}^{H} \frac{\bar{u}^{2}}{4 D^{2}}\left[\frac{\frac{x^{2}}{D^{2}}}{\left(\frac{x}{D}\right)^{2}+1}-\frac{2 x}{D \sqrt{\left(\frac{x}{D}\right)^{2}+1}}+1\right] d x$

On integrating $(9 b)$ we get

$$
\begin{gathered}
E_{d}=\frac{L h G}{4} \frac{\bar{u}^{2}}{D^{2}} \times \\
\times\left[2 H-D \arctan \frac{H}{D}-2 D\left(\sqrt{\frac{H^{2}}{D^{2}}+1}-1\right)\right] .
\end{gathered}
$$

Calculation shows that the expression in square brackets in (9b) is $0.43 D$ given (6), whatever is the value of $H$. We thus have

$$
\begin{aligned}
E_{d} & =\frac{0.1075}{4} L h G \bar{u}^{2} \\
\text { or } E_{d} & =0.1075 \frac{1-\alpha^{2}}{2 \alpha} \frac{L h G \bar{u}^{2}}{H} .
\end{aligned}
$$

Comparison of this value with that of $E_{d}$ from (12) shows that the two are exactly equal for $\alpha=0.17(H=2.86 D)$. When $\alpha=0.15$, the difference between the values of $E_{d}$ from (12) and (12a) is $13 \%$ toward lesser values.

Assuming the simplest linear relationship for the decaying slip function $u(x)$ in the form

$$
u(x)=\frac{\bar{u}}{2}\left(1-\frac{x}{H}\right)
$$

we get for potential energy $E_{d}$ from (9)

$$
E_{d}=\frac{L h G \bar{u}^{2}}{4 H} \text { or } E_{d}=0.25 \frac{L h G \bar{u}^{2}}{H},
$$

which is different from the value of $E_{d}$ based on (12) by a mere $15 \%$. To sum up, with fixed $\bar{u}$ and $H$, the character of the decaying slip function $u(x)$ is not significant for determining the value of potential energy.

We shall use (12) to find $E_{d}$ for the 1988 Spitak, Armenia earthquake with the following parameters [13]:

$$
\begin{gathered}
L=38 \mathrm{~km}=38 \times 10^{3} \mathrm{~m}, \\
h=11 \mathrm{~km}=11 \times 10^{3} \mathrm{~m}, \\
u_{\max }=2 \mathrm{~m}=200 \mathrm{~cm}, \\
\bar{u}=1.22 \mathrm{~m}=122 \mathrm{~cm} . \\
\mathrm{H}=10^{3} \times(5 \times 1.22+15)= \\
=21.1 \mathrm{~km}=21.1 \times 10^{3} \mathrm{~m} \\
G=5 \times 10^{11} \mathrm{dyne} / \mathrm{cm}^{2}=10^{16} \mathrm{~Pa} \\
E_{d}=\frac{9.87 \times 38 \times 10^{5} \times 11 \times 10^{5} \times 5 \times 10^{11} \times(122)^{2}}{\quad 32 \times 21.1 \times 10^{5}}= \\
=454726 \times 10^{16} \mathrm{dyne} \cdot \mathrm{cm} \\
\text { or } \quad E_{d}=0.45 \times 10^{22} \mathrm{ergs}=0.45 \times 10^{15} \mathrm{~J} .
\end{gathered}
$$

The energy class of an earthquake $k_{d}$ as found from strain energy $E_{\mathrm{d}}$ will be

$$
k_{d}=\log E_{d}-7 .
$$

Substituting the value of $E_{d}$ for the Spitak earthquake, we get

$$
k_{d}=\log 0.45+22-7=-0.34+15=14.66 \text {. }
$$

For comparison purposes we now find the value of $k_{S}$ from magnitude $M_{S}$ by transforming (1) to the form

$$
\begin{aligned}
& k_{S}=\log E_{S}=11.8+1.5 M_{S}-7= \\
& =1.5 M_{S}+4.8 .
\end{aligned}
$$

For the Spitak earthquake, whose magnitude was $M_{S}=$ 6.8 , we find

$$
k_{S}=1.5 \times 6.8+4.8=15 \text {. }
$$

We thus see that the difference in energy class as found from (13) and (1a) is inconsiderable. In a similar way for 44 large earthquakes we found $k_{d}$ and $k_{S}$ from (13) and (1a) (Table 2). All the data used for Table 2 were borrowed from [13] who give macro seismic parameters for the rupture zones of 244 large earthquakes for the period 1853-1993. We only used those earthquakes for which all the three parameters $(L, h, \bar{u})$ were available in their paper, because we could then find energy using (12). The main inference from our results is that the values of energy found from (12) generally obey the well- known relationships that specify that the value of released energy increases with increasing extent of surface rupture $L$, rupture depth $h$, and relative slip of paired blocks $\bar{u}$. These relationships are plotted in Fig. 3. In the same figure are shown also indirect depen- 
dences $k_{d}$ from the value of area of plane of rupture $F=L h$, volume of strain space around the rupture $V=L h H$ and value of seismic moment $M_{0}=F G \bar{u} \quad(F$ in $\mathrm{cm}^{2}, V$ in $\mathrm{cm}^{3}, M_{0}$ in dyne $\left.\mathrm{cm}\right)$. The respective formulas are as follows ( $E_{d}$ in Joules, $L$ and $h$ in $10^{3} \mathrm{~m}$, and $\bar{u}$ in $\mathrm{m}$ ):

$$
\begin{aligned}
\log E_{d} & =2.74 \log L+10.04 \\
\log E_{d} & =2.46 \log \bar{u}+14.62 \\
\log E_{d} & =0.19 h+11.89 \\
\log E_{d} & =1.79 M_{S}+2.16 \\
\log E_{d} & =2.15 \lg F-12.83 \\
\log E_{d} & =1.88 \lg V-21.36 \\
\log E_{d} & =1.35 M_{0}-21.14
\end{aligned}
$$

It can be seen from the last plot in Fig. 3 that the energy classes of the earthquakes under consideration as found from (12) and (13) proposed here for use and the respective magnitudes $M_{S}$ (Table 2 ) are linearly related:

$$
k_{d}=1.79 M_{S}+2.16
$$

This shows that earthquake energy linearly depends on earthquake magnitude using both the method for energy determination we propose in this paper and that from (1a). The mean deviation $k_{S}-k_{d}$ between (1a) and (1b) does not exceed 0.46 .

It can be seen from Table 2 that the energy class values for most earthquakes, except for 3-5 events whose relative slip $\bar{u}$ was below $0.2 \mathrm{~m}$, which were found from (13) and (1a) are of the same order. The mean deviation of these is 0.66 (Fig. 4a). As expected, some slight deviation occurs for small earthquakes whose mean slip was $\bar{u}<0.2 \mathrm{~m}$; when these events were excluded from this calculation, the mean deviation becomes about 0.5 , that is, the scatter does not exceed one energy class unit. Since both formulas are approximate and semi-empirical, the difference is only

\begin{tabular}{|c|c|c|c|c|c|c|c|c|c|c|c|c|}
\hline & Country & $\begin{array}{l}\text { Earthquake } \\
\text { location }\end{array}$ & $\begin{array}{l}\text { Date } \\
\text { of earthquake } \\
\text { occurrence }\end{array}$ & $\begin{array}{l}\text { Type } \\
\text { of slip }\end{array}$ & $\begin{array}{l}\text { Earthquake } \\
\text { magnitude, } \\
\text { Ms }\end{array}$ & $\begin{array}{l}\text { Rupture } \\
\text { length } \\
\text { L (km) }\end{array}$ & $\begin{array}{l}\text { Rupture } \\
\text { depth } \\
\text { h (km) }\end{array}$ & $\begin{array}{l}\text { Maximum } \\
\text { slip } \\
\operatorname{umax}(\mathbf{m})\end{array}$ & $\begin{array}{l}\text { Mean } \\
\text { slip } \\
\bar{u}_{(\mathrm{m})}\end{array}$ & $\begin{array}{l}\text { Value of } \\
H \text { from } \\
\text { (3) }(\mathrm{km})\end{array}$ & $\begin{array}{l}\text { Energy } \\
\text { classes } k_{d} \\
\text { based on } \\
E_{d}, \\
\text { using (13) }\end{array}$ & $\begin{array}{l}\text { based } \\
\text { on } M_{s} \text {, } \\
\text { using } \\
\text { (la) }\end{array}$ \\
\hline 1 & $\begin{array}{l}\text { United } \\
\text { States }\end{array}$ & Fort Tejon & 09.01 .1857 & RL & 8.3 & 297 & 12 & 9.4 & 6.4 & 50.84 & 16.68 & 17.25 \\
\hline 2 & $\begin{array}{l}\text { United } \\
\text { States }\end{array}$ & Owens Valley & 26.03 .1872 & RL-N & 8.0 & 108 & 15 & 11 & 6.0 & 45 & 16.30 & 16.8 \\
\hline 3 & Japan & Nobi & 27.10 .1891 & LL & 8.0 & 80 & 15 & 8.0 & 5.04 & 40.25 & 16.06 & 16.8 \\
\hline 4 & Japan & Rikuu & 31.08 .1896 & $\mathrm{R}$ & 7.2 & 40 & 21 & 4.4 & 2.59 & 27.95 & 15.49 & 15.6 \\
\hline 5 & $\begin{array}{l}\text { United } \\
\text { States }\end{array}$ & San Francisco & 13.01.1906 & $\mathrm{RL}$ & 7.8 & 432 & 12 & 6.1 & 3.3 & 31.5 & 16.44 & 16.5 \\
\hline 6 & $\begin{array}{l}\text { United } \\
\text { States }\end{array}$ & $\begin{array}{l}\text { Pleasant } \\
\text { Valley }\end{array}$ & 03.10 .1915 & $\mathrm{~N}$ & 7.6 & 62 & 15 & 5.8 & 2.0 & 25 & 15.36 & 16.2 \\
\hline 7 & China & Kansy & 16.12 .1920 & LL & 8.5 & 220 & 20 & 10.0 & 7.25 & 51.25 & 16.84 & 17.55 \\
\hline 8 & Japan & North Izu & 25.11 .1930 & LL- R & 7.3 & 35 & 12 & 3.8 & 2.9 & 29.5 & 15.26 & 15.75 \\
\hline 9 & China & Kehetuohai & 10.08 .1931 & $\mathrm{RL}$ & 7.9 & 180 & 20 & 14.6 & 7.38 & 51.9 & 16.76 & 16.65 \\
\hline 10 & Turkey & Erzihcan & 26.12 .1939 & $\mathrm{RL}$ & 7.8 & 360 & 20 & 7.5 & 1.85 & 24.25 & 16.19 & 16.5 \\
\hline 11 & $\begin{array}{l}\text { United } \\
\text { States }\end{array}$ & $\begin{array}{l}\text { Imperial } \\
\text { Valley }\end{array}$ & 19.05 .1940 & RL & 7.2 & 60 & 11 & 5.9 & 1.5 & 22.5 & 15.01 & 15.6 \\
\hline 12 & China & Damxung & 18.11 .1951 & RL & 8.0 & 200 & 10 & 12.0 & 8.0 & 65 & 16.55 & 16.8 \\
\hline 13 & $\begin{array}{l}\text { United } \\
\text { States }\end{array}$ & Dixie Valley & 16.12 .1954 & RL-R & 6.8 & 45 & 14 & 3.8 & 2.1 & 25.5 & 15.22 & 15.0 \\
\hline 14 & Turkey & Abant & 26.05 .1957 & RL & 7.0 & 40 & 8 & 1.65 & 0.55 & 17.75 & 13.92 & 15.3 \\
\hline
\end{tabular}
natural.

One slip component only was used when deriving (12). From Table 2 (the Rupture type column) it can be seen that for some earthquakes there was a more complex slip kinematics; hence, the energy values for such earthquakes as found from (12) will be the least.

Table 2. Earthquake parameters and the energy $k d$ and $k S$ as calculated from (13) and (1a) 


\begin{tabular}{|c|c|c|c|c|c|c|c|c|c|c|c|c|}
\hline 15 & Mongolia & Gobi-Altai & 04.12 .1957 & LL & 7.9 & 300 & 20 & 9.6 & 6.54 & 47.7 & 16.92 & 16.65 \\
\hline 16 & $\begin{array}{l}\text { United } \\
\text { States }\end{array}$ & Hebgen Lake & 18.08 .1959 & $\mathrm{~N}$ & 7.6 & 45 & 17 & 6.1 & 2.14 & 25.7 & 15.32 & 16.2 \\
\hline 17 & Iran & Dasht-e-Bayaz & 31.08 .1968 & LL & 7.1 & 110 & 20 & 5.2 & 2.3 & 26.5 & 15.83 & 15.45 \\
\hline 18 & Turkey & Gediz & 28.03 .1970 & $\mathrm{~N}$ & 7.1 & 63 & 17 & 2.8 & 0.86 & 19.3 & 14.80 & 15.45 \\
\hline 19 & $\begin{array}{l}\text { United } \\
\text { States }\end{array}$ & San Fernando & 09.02.1971 & R-LL & 6.5 & 17 & 14 & 2.5 & 1.5 & 22.5 & 14.56 & 13.75 \\
\hline 20 & China & Luhuo & 06.02 .1973 & LL & 7.3 & 110 & 13 & 3.6 & 1.3 & 21.5 & 15.24 & 15.75 \\
\hline 21 & Guatemala & Motagua & 04.02 .1976 & LL & 7.5 & 257 & 13 & 3.4 & 2.6 & 28.0 & 16.09 & 16.05 \\
\hline 22 & Turkey & Caldiran & 24.11 .1976 & $\mathrm{RL}$ & 7.3 & 90 & 18 & 3.5 & 2.05 & 25.25 & 15.62 & 15.75 \\
\hline 23 & Iran & Bob-Tangol & 19.12.1977 & $\mathrm{RL}$ & 5.8 & 14 & 12 & 0.3 & 0.12 & 15.6 & 12.38 & 13.5 \\
\hline 24 & Greece & Thezzaloniki & 20.06 .1978 & $\mathrm{~N}$ & 6.4 & 28 & 14 & 0.22 & 0.08 & 15.4 & 12.40 & 14.4 \\
\hline 25 & Iran & $\begin{array}{l}\text { Ta- } \\
\text { bas-e-Colshan }\end{array}$ & 16.09 .1978 & $\mathrm{R}$ & 7.5 & 74 & 22 & 3.0 & 1.5 & 22.5 & 15.39 & 16.05 \\
\hline 26 & $\begin{array}{l}\text { United } \\
\text { States }\end{array}$ & $\begin{array}{l}\text { Homestead } \\
\text { Valley }\end{array}$ & 15.03 .1979 & $\mathrm{RL}$ & 5.6 & 6 & 4 & 0.1 & 0.05 & 15.25 & 10.78 & 13.2 \\
\hline 27 & Australia & Cadoux & 02.06 .1979 & $\mathrm{R}$ & 6.1 & 16 & 6 & 1.5 & 0.5 & 17.5 & 13.32 & 13.95 \\
\hline 28 & $\begin{array}{l}\text { United } \\
\text { States }\end{array}$ & El Centro & 15.10 .1979 & $\mathrm{RL}$ & 6.7 & 51 & 12 & 0.8 & 0.18 & 15.9 & 13.28 & 14.85 \\
\hline 29 & Iran & Koli & 27.11 .1979 & LL-R & 7.1 & 75 & 22 & 3.9 & 1.2 & 21.0 & 15.24 & 15.45 \\
\hline 30 & Algeria & El Asman & 10.10 .1980 & $\mathrm{R}$ & 7.3 & 55 & 15 & 6.5 & 1.54 & 22.7 & 15.12 & 15.75 \\
\hline 31 & Italy & $\begin{array}{l}\text { South } \\
\text { Apennines }\end{array}$ & 23.11 .1980 & $\mathrm{~N}$ & 6.9 & 60 & 15 & 1.15 & 0.64 & 18.2 & 14.49 & 15.15 \\
\hline 32 & Greece & Corinth & 25.02 .1981 & $\mathrm{~N}$ & 6.4 & 19 & 16 & 1.5 & 0.6 & 18.0 & 13.97 & 14.4 \\
\hline 33 & Greece & Corinth & 04.03 .1981 & $\mathrm{~N}$ & 6.4 & 26 & 18 & 1.1 & 0.6 & 18.0 & 14.16 & 14.4 \\
\hline 34 & $\begin{array}{l}\text { United } \\
\text { States }\end{array}$ & Borah Peak & 28.10 .1983 & N-LL & 7.3 & 33 & 20 & 2.7 & 0.8 & 19.0 & 14.53 & 15.75 \\
\hline 35 & Algeria & Constantine & 27.10 .1985 & LL & 5.9 & 21 & 13 & 0.12 & 0.1 & 15.5 & 12.43 & 13.65 \\
\hline 36 & Australia & Marryat Creek & 30.03 .1986 & R-LL & 5.8 & 13 & 3 & 1.3 & 0.5 & 17.5 & 12.93 & 13.5 \\
\hline 37 & Greece & Kalamata & 13.09 .1986 & $\mathrm{~N}$ & 5.8 & 15 & 14 & 0.18 & 0.15 & 15.75 & 12.66 & 13.5 \\
\hline 38 & $\begin{array}{l}\text { New } \\
\text { Zealand }\end{array}$ & Edgecumbe & 02.03 .1987 & $\mathrm{~N}$ & 6.6 & 32 & 14 & 2.9 & 1.7 & 23.5 & 14.93 & 14.7 \\
\hline 39 & $\begin{array}{l}\text { United } \\
\text { States }\end{array}$ & $\begin{array}{l}\text { Superstition } \\
\text { Hills }\end{array}$ & 24.11 .1987 & $\mathrm{RL}$ & 6.6 & 30 & 11 & 0.92 & 0.54 & 17.5 & 13.92 & 14.7 \\
\hline
\end{tabular}




\begin{tabular}{|c|c|c|c|c|c|c|c|c|c|c|c|c|}
\hline 40 & Australia & $\begin{array}{l}\text { Tennant } \\
\text { Greek }\end{array}$ & 22.01 .1988 & $\mathrm{R}$ & 6.3 & 13 & 9 & 1.3 & 0.63 & 18.15 & 13.59 & 14.25 \\
\hline 41 & China & $\begin{array}{l}\text { Lancand } \\
\text { Gengma }\end{array}$ & 06.11 .1988 & RL & 7.3 & 80 & 20 & 1.5 & 0.7 & 18.5 & 14.81 & 15.75 \\
\hline 42 & Armenia & Spitak & 07.12 .1988 & R-RL & 6.8 & 38 & 11 & 2.0 & 1.22 & 21.1 & 14.65 & 15.0 \\
\hline 43 & Canada & Ungava & 25.12.1989 & $\mathrm{R}$ & 6.3 & 10 & 5 & 2.0 & 0.8 & 19.0 & 13.41 & 14.25 \\
\hline 44 & $\begin{array}{l}\text { United } \\
\text { States }\end{array}$ & Landers & 28.06.1992 & $\mathrm{RL}$ & 7.6 & 62 & 12 & 6.0 & 2.95 & 29.75 & 15.52 & 16.2 \\
\hline
\end{tabular}

Note: $R L$ denotes right lateral strike slip, LL left lateral slip, $R$ reverse movement, and $N$ normal faulting. The true earthquake energy is $E=10^{k} J$

It was noted above that the depth of felt strain $H$ can also be found from (6) through fault depth $h$. We used the values of $h$ given in Table 2 to find new values of $H$ for all 44 earthquakes and then substituted them into (12a) and (13) to find the corresponding values of $E_{d}$ and $k_{d}$.
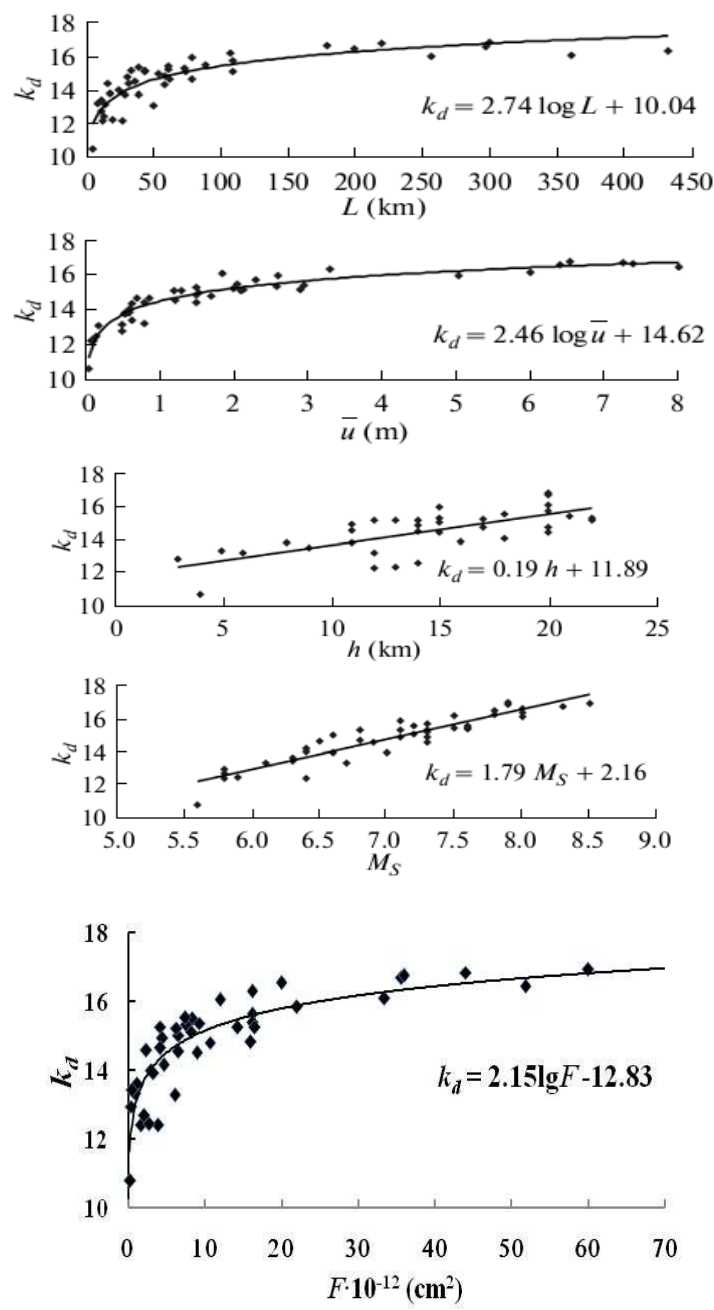
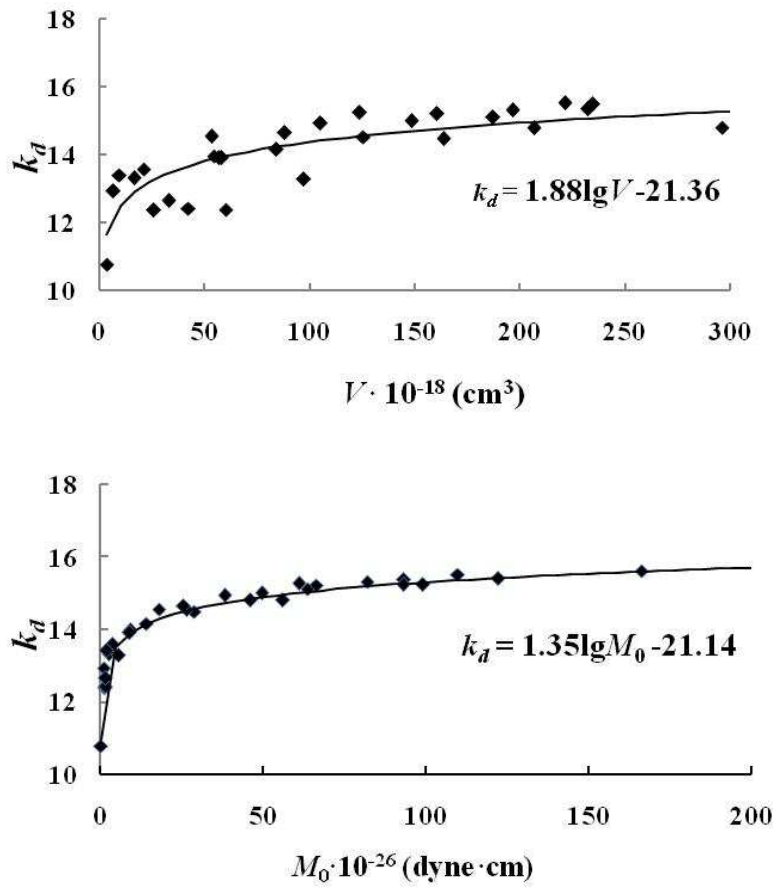

Fig. 3. Relationships among earthquake energy class, rupture length, relative slip, and earthquake magnitude (based on data from $4 \underline{4}$ earthquakes). $k d$ is energy class defined by (13), L rupture length, $\bar{u}$ relative slip, $h$ rupture depth, and Ms earthquake magnitude as given in Table 2, F area of plane of rupture, $V$ volume of strain space, MO seismic moment.

For the coefficient $\alpha=0.2$ the difference in energy class as found from values of $H$ according to (3) and (6) is shown in Fig. 4b. One can see that the mean deviation of energy classes does not exceed 0.2 .

We now discuss another comparative analysis. Kasahara notes in his book that knowledge of stress drop $\Delta \tau$ can be used to estimate strain energy $E_{d}$ based on known rupture length. The book does not contain any formula for finding $E_{d}$, but the appendix contains, for 6 earthquakes out of 43 , along with the earthquake rupture parameters, the associated energies based on seismic waves $E_{S}$ and the change in strain energy $E_{d}$ on rupturing. For three of the earthquakes we consider (nos. 5, 8, and 11, see Table 2), these parameters and the corresponding energy classes $k_{S}$ and $k_{d}$ 
as found from $E_{S}$ and $E_{d}$ using (1a) and (13) and from Kasahara's data are listed in Table 3; it can be seen that the results for two earthquakes ( 8 and 11 ) are fairly consistent. As to earthquake no. 5 , the difference in $k_{S}$ between the two results is due to the considerable difference in earthquake magnitude $M_{S}$ (8.25 and 7.8, respectively); for $k_{d}$ the cause lies in the nearly double difference between mean slips $\bar{u}$ at the rupture $(6.1 \mathrm{~m}$ and $3.3 \mathrm{~m}$, respectively).
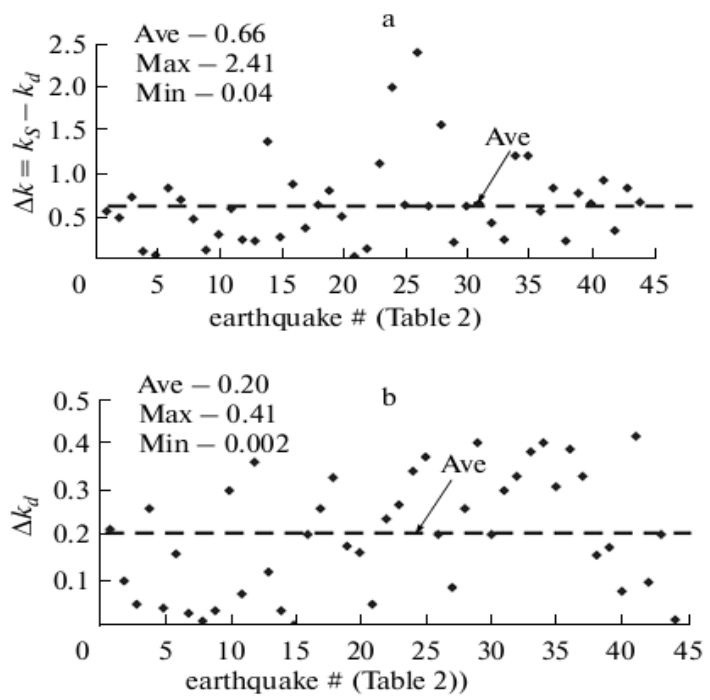

Fig. 4. Differences between the earthquake energy classes derived from earthquake magnitude and strain energy calculated by several methods. (a) energy class differences $\Delta k=k_{S}-k_{d}$ based on magnitude $\mathrm{M}_{\mathrm{s}}$ from (1a) and based on strain energy $\mathrm{E}_{\mathrm{d}}$ using (13) for 44 earthquakes and the respective means (dashed line); (b) energy class differences $\Delta k_{d}$ calculated from strain energy $\mathrm{E}_{\mathrm{d}}$ using (12) and (12a) for $\alpha=0.2$ for 44 earthquakes and the mean value (dashed line).

Table 3. Comparative analysis of energy classes

\begin{tabular}{|c|c|c|c|c|c|c|c|c|c|}
\hline $\begin{array}{l}\text { For \#\# se } \\
\text { Ta-ble } 2\end{array}$ & Earthquake & & $\boldsymbol{M}_{s}$ & $L, 10^{-3} \mathrm{~m}$ & $h, 10^{-3} \mathrm{~m}$ & $u_{\max , \mathbf{m}}$ & $\bar{u}, \mathbf{m}$ & $\begin{array}{l}\text { Energy class } \\
\text { magnitude- } \\
\text { based } k_{S}\end{array}$ & $\begin{array}{l}\text { based on } \\
\text { potential energy } k_{d}\end{array}$ \\
\hline \multirow{2}{*}{5} & San-Francisco & $\mathrm{a}$ & 8.25 & 430 & 15 & - & $5-7$ & 17.3 & 17.47 \\
\hline & 18.04 .1906 & $\mathrm{~b}$ & 7.8 & 432 & 12 & 6.1 & 3.3 & 16.5 & 16.44 \\
\hline \multirow{2}{*}{8} & \multirow{2}{*}{ North Izu 25.11.1930 } & a & 7.1 & 20 & 11 & - & 3.0 & 15.3 & 15.47 \\
\hline & & $\mathrm{b}$ & 7.3 & 35 & 12 & 3.8 & 2.9 & 15.75 & 15.26 \\
\hline \multirow{2}{*}{11} & Imperial & $\mathrm{a}$ & 7.1 & 70 & 11 & - & 2.0 & 15.0 & 15.17 \\
\hline & Valley 19.05 .1940 & $\mathrm{~b}$ & 7.2 & 60 & 11 & 5.9 & 1.5 & 15.6 & 15.01 \\
\hline
\end{tabular}

Note: (a) based on [4]. (b) based on [13] using (1a) and the formulas (12) and (13) here proposed. The values of $\mathrm{u}_{\max }$ are not given by Kasahara [4]. The true earthquake energy is $E=10^{k} \mathrm{~J}$.

\section{Strain Energy at the Rupture}

There is one issue that is frequently debated in the literature concerned with the amount of earthquake energy, viz., what is the part of the total energy released by an earthquake that concentrates in the rupture zone [1]. It is natural to assume that the bulk of the energy is released just in the rupture zone, because it is in the rupture zone that the tangential stresses exceed the strength everywhere. In the simplest possible case where the strain field contains a single shear component $\gamma_{x y}$ (as is the case in all calculations above), the energy in the medium per unit volume is given by [4]

$$
e=\frac{1}{2} G \gamma_{T}^{2},
$$

where $\gamma_{T}$ is the shear strength limit for the crustal rocks. By various estimates $[4,14,16]$, the strength limit $\gamma_{T}$ varies in the range $\gamma_{T}=(1 \div 2) \times 10^{-4}$. The Earth's crust deforms as far as the limit in an elastic manner, but never surpasses it without fracture. According to several specialists $[4,14,16]$, that value of $\gamma$ is considerably below those given by laboratory experiments, where one gets $\gamma_{T}=10^{-3}$. This contradiction can be explained by the fact that the actual crust contains numerous cracks that reduce the macroscopic strength of crustal rocks. As K. Kasahara wrote, “....any chain cannot be stronger than its weakest link". 
Assuming $G=5 \times 10^{11} \mathrm{dyne} / \mathrm{cm}^{2}=5 \times 10^{16} \mathrm{~Pa}$, $\gamma_{T}=1.1 \times 10^{-4}$, K. Kasahara used (15) to get

$$
e \cong 3000 \mathrm{ergs} / \mathrm{cm}^{3}=300 \mathrm{~J} / \mathrm{m}^{3} \text {. }
$$

It follows that all of the potential energy that was being accumulated near the rupture plane with unit width $\Delta H=10^{-2} \mathrm{~m}$ will be, according to our formulation ( $L$ and $h$ are in $10^{-2} \mathrm{~m}$ ):

$$
E_{f}=L \cdot h \cdot 1 \cdot e=3000 L h \text { ergs, }
$$

and the corresponding energy class will be

$$
k_{f}=\log E_{f}-7 .
$$

We substituted the values of $L$ and $h$ from Table 2 into (16a) to find the strain energy in the rupture zone $E_{f}$ for 44 earthquakes and the corresponding energy classes $k_{f}$. A graphic representation of the ratios between energy classes in the rupture zone $k_{f}$ and those based on the entire energy in the medium $k_{d}$ (according to Table 2) is shown in Fig. 5, where one can see that the average value of $k_{f} / k_{d}$ is 0.63 .

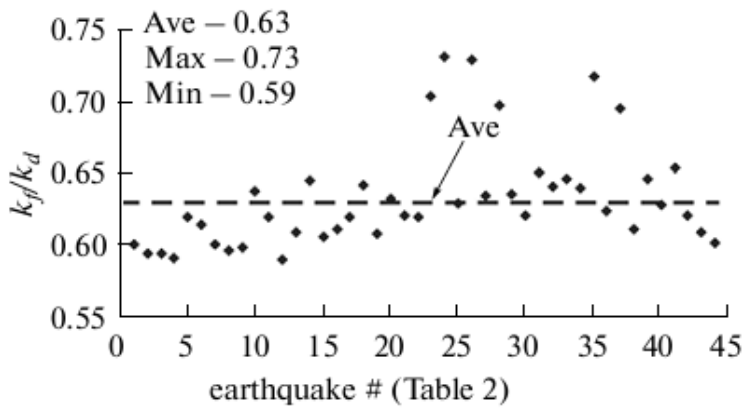

Fig. 5. Ratios of energy class based on strain energy in the rupture zone $k f$ using relation (17) to the energy classes $k$ for the entire deformable medium using relation (12) for $\Delta H=10^{-2} \mathrm{~m}$ for 44 earthquakes and the mean value (dashed line).
The post seismic ruptures do not usually form a single geometrically regular plane but make a network of several segments spaced at a definite distance $\Delta H$ perpendicularly to the fault. This distance can provisionally be assumed to be the fault width; hence, one can accept that the pre seismic strain has reached the strength limit $\gamma_{T}$ in the entire volume $L \cdot h \cdot \Delta H$ in the earth (the rupture zone), so that the potential energy in the rupture zone will be

$$
\begin{aligned}
& E_{f}=3000 \cdot L \cdot h \cdot \Delta H \text { egrs } \\
& =3000 \cdot L \cdot h \cdot \Delta H \times 10^{-7} \mathrm{~J} .
\end{aligned}
$$

Actually, the energy class $k_{f}$ in the rupture zone will therefore be considerably above the value indicated $\left(0.63 k_{d}\right)$ for $\Delta H=10^{-2} \mathrm{~m}$. Table 4 lists mean values of the energy characteristics, $k_{f} / k_{d}, k_{d}-k_{f}$ and $E_{d} / E_{f}$ (based on 44 earthquakes), which were calculated by the method outlined above for values of $\Delta H$ between $10^{-2} \mathrm{~m}$ and $10^{3} \mathrm{~m}$. It can be seen from this table that, depending on the provisional fault width $\Delta H$, the amount of energy released in the rupture zone rather rapidly increases with increasing $\Delta H$ and reaches $35 \%$ of the total earthquake energy released for $\Delta H=10^{3} \mathrm{~m}$.

The aftershock process is due to some fraction of the strain energy remaining in the earth outside the rupture zone. The field of stored strain (stress) before an earthquake usually has a very complex structure related to an inhomogeneous distribution of the strength and density of the rocks and the presence of cracks and ruptures caused by previous earthquakes [16]. For this reason the main shock will trigger subsequent aftershocks (in weaker structures) until a new state of equilibrium is reached by the medium that had been deformed during the pre seismic period. It would be natural to expect that the aftershocks will mostly occur along the main shock rupture and around it where the stresses pri-

\begin{tabular}{|c|c|c|c|c|c|c|}
\hline \multirow{2}{*}{ Mean values } & \multicolumn{6}{|c|}{ Provisional rupture width $\Delta H$ in $\mathbf{m}$} \\
\hline & $\overline{10^{-2}}$ & $10^{-1}$ & $10^{0}$ & $10^{1}$ & $10^{2}$ & $10^{3}$ \\
\hline$k_{f} / k_{d}$ & 0.63 & 0.70 & 0.77 & 0.84 & 0.91 & 0.97 \\
\hline$k_{d}-k_{f}$ & 5.46 & 4.46 & 3.46 & 2.46 & 1.46 & 0.46 \\
\hline$E_{d} / E_{f}$ & 288000 & 28800 & 2880 & 288 & 28.8 & 2.88 \\
\hline
\end{tabular}
marily concentrated. A large number of aftershocks will be an indirect indication that there are many variously weakened rock portions around the main rupture.

Table 4. Mean energy characteristics in relation to rupture width (based on data from 44 earthquakes, see Table 2)

\section{Results}

This paper presents a simple way of estimating the potential strain energy stored in the earth around a future earthquake rupture based on the macro seismic consequences of the earthquake: rupture length, rupture depth, and mean slip along the fault. The method we propose is based on representation of a volume in a pre stressed medium as the volumes of two identical parallelepipeds that are situated on both sides of the future rupture line and are subject to pure shear. The sides of these parallelepipeds are assumed to be rupture length $L$, rupture depth $h$, and the distance $H$ in the direction perpendicular to the rupture, beyond which distance the earth can be assumed to be free of the stresses associated with the impending earthquake. It is recom- 
mended that we determine the value of $H$ after the occurrence of the earthquake from mean slip $\bar{u}$ using the empirical formula (3) or from rupture depth $h$ using the theoretical formula (6). These simplifications reduce the general expression for potential energy (2) to (12) or (12a), respectively. We demonstrate that the energy classes calculated from these formulas differ little, viz., by 0.2 units on the average. The parameters of 44 earthquakes were used to find their respective energy classes using (12) and (13), with the results being displayed in Table 2 . In this table one also finds the energy classes of the same earthquakes found by determining the energy of seismic waves from earthquake magnitude using (1a). Comparative analysis shows that the mean deviation between these energy classes is 0.46 . It is found that strain energy is little affected by how slip decays perpendicularly to the fault with given $\bar{u}$ and $H$.

The method outlined above can be used to assess the energy that is liberated around the rupture in relation to the provisional rupture width $\Delta H$. Comparative assessment of the ratio between the strain energy in the rupture zone $E_{f}$ and the total strain energy $E_{d}$ showed that more than $35 \%$ of the total strain energy is stored in the zone of the future rupture when the rupture width is $10^{3} \mathrm{~m}$.

\section{References}

[1] J.N. Brune, The Physics of Earthquake Strong Motion, in Lomnitz, C. and Rosenblueth, E., Eds., Seismic Risk and Engineering Decisions, New York: Elsevier Sci. Publ. Co., 1976, pp.141-177.

[2] C. Lomnitz, and K.S. Singh, Earthquakes and Earthquake Prediction, in Lomnitz, C. and E. Rosenblueth, , Eds., Seismic Risk and Engineering Decisions, New York: Elsevier Sci. Publ. Co., 1976, pp. 3-30
[3] J. Rice, Ed., The Mechanics of Earthquake Rupture, Amsterdam: Elsevier, 1982.

[4] K. Kasahara, Earthquake Mechanics, Cambridge University Press, 1981.

[5] S.S. Grigoryan, On the Mechanics of Earthquake Generation and the Meaning of Empirical Relations in Seismology, Dokl. ANSSSR, 1988, vol. 299, no. 5, pp. 1094-1101.

[6] Ch. Richter, Elementary Seismology, San Francisco: W.H. Freeman and Company, 1958.

[7] B. Gutenberg, and C.F. Richter, Earthquake Magnitude, Intensity, and Acceleration, Bull. Seismol. Soc. Amer., 1956, vol. 46 , no. 2 , pp. 105-145.

[8] H. Jeffreys, The Earth, Cambridge University Press, 1970.

[9] N.N. Ambraseys, MaximUm Intensity of GroUnd Movements Caused by Faulting, Proc. 4th World Conf. Earthq. Eng., vol. 1, A-2, pp. 154-171, Santiago, Chile, 1969.

[10] D. McKenzie, and J.N. Brune, Melting on Fault Planes during Large Earthquakes, Geophys. J. R. Astr. Soc., 1972, vol. 29 , pp. 65-78.

[11] S.P. Timoshenko, and J. Goudier, Theory of Elasticity. 2nd ed., New York: McGraw-Hill, 1951.

[12] H.F. Reid, The Elastic Rebound Theory of Earthquakes, Univ, Calif. Publ. Bull. Dept. Geol., 1911, no.6.

[13] D.L. Wells, and K.I. Coppersmith, New Empirical Relationship among Magnitude, Rupture Length, Rupture Width, Rupture Area, and Surface Displacement, Bull. Seismol. Soc. Amer., 1994, vol. 84, no. 4, pp. 974-1002.

[14] T. Rikitake, Earthquake Prediction, Amsterdam: Elsevier, 1976.

[15] L. Knopoff, Energy Release in Earthquakes, Geophys. J., 1958, vol. 1, no. 1, pp. 44-52.

[16] K. Mogi, Earthquake Prediction, Academic Press, 1985. 